\title{
PROBABILIDADE DE ABANDONO DE TERAPÊUTICA DIETÉTICA PARA PERDA DE PESO: O PAPEL DE FATORES PSICOSSOCIAIS
}

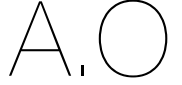

ARTIGO ORIGINAL

1 Programa de Pós-

Graduação Interunidades em Nutrição Humana

Aplicada da Universidade

de São Paulo,

Avenida Nova Cantareira,

n. ${ }^{\circ} 2445$, ap. 43

São Paulo, Bras

2 Faculdade de Saúde

Pública da Universidade de

São Paulo,

Avenida Doutor Arnaldo,

$0^{\circ} 715$,

São Paulo, Brasil

${ }^{3}$ Fundação Instituto de Pesquisas Econômicas - FIPE,

Av. Corifeu de Azevedo

Marques, n. ${ }^{\circ} 5677$,

São Paulo, Bras

${ }^{4}$ Faculdade de

Economia, Administração

e Contabilidade da

Universidade de São

Paulo, Avenida Engenheiro

Antônio Eiras Garcia,

n. ${ }^{\circ}$ 09, ap. 113, Bloco Entry,

São Paulo, Brasi

${ }^{5}$ Departamento de

Economia da Faculdade de

Economia, Administração

e Contabilidade da

Universidade de São

Paulo,

Av. Prof. Luciano

Gualberto, n. ${ }^{\circ} 908$

São Paulo, Brasil

tEndereço para correspondência:

Luciana Alves Cangeran

Santiago

Avenida Nova Cantareira,

n. 2445 , apartamento 43 ,

São Paulo, Brasil

ucianacan@outlook.com

Histórico do artigo:

Recebido a 31 de agosto de 2020 Aceite a 18 de dezembro de 2020

\author{
PROBABILITY OF ABANDONING DIETARY THERAPY FOR \\ WEIGHT LOSS: THE ROLE OF PSYCHOSOCIAL FACTORS
}

Luciana Alves Cangerana Santiago" ${ }^{*}$; Ana Maria Cervato-Mancuso²; Jackson William Rosalino ${ }^{3}$; Raí da Silva Chicoli ${ }^{4}$; Denise Cavallini Cyrillo ${ }^{5}$

\section{RESUMO}

INTRODUÇÃo: O excesso de peso vem alcançando proporções epidémicas mundiais, e com ele emergem outras doenças crónicas não transmissíveis. Nesse contexto ocorre um aumento pela procura por terapêutica dietética para perda de peso, nem sempre bem-sucedida em função de baixa adesão e consequente abandono dessas terapêuticas.

OBJETIVOS: Investigar a probabilidade de abandono de terapêutica dietética para perda de peso segundo aspetos psicossociais, socioeconómicos, demográficos, antropométricos e clínicos.

METODOLOGIA: Estudo transversal quantitativo retrospetivo. Desenvolvido em duas instituições de saúde e uma de ensino. Incluídos adultos de 20 a 60 anos que já tivessem se submetido à terapêutica dietética para a perda de peso. Foram aplicados questionários para coleta de dados psicossociais, socioeconómicos, demográficos, antropométricos e clínicos. Com base nos dados coletados, estimou-se um modelo utilizando o método Probit de regressão múltipla para investigar quais variáveis influenciam a probabilidade de abandono da terapêutica dietética para perda de peso.

RESULTADOS: A amostra foi composta por 86 indivíduos, sendo que 57\% haviam abandonado terapêutica dietética para perda de peso. A estimação do modelo mostrou que a variável escolaridade aumenta em 18,9 pontos percentuais o abandono de terapêutica dietética para perda de peso. Em relação aos fatores psicossociais o aumento de um grau na satisfação com a terapêutica e no suporte social reduz a probabilidade de abandono em 7,8 pontos percentuais e 5,7 pontos percentuais, respetivamente. E as variáveis de caráter clínico, económico, demográfico e antropométrico não se apresentaram estatisticamente significativas.

CONCLUSÕES: Verificou-se que alto nível de escolaridade favorece o abandono de terapêutica dietética para perda de peso e a satisfação e suporte social atuam de forma contrária reduzindo o abandono, neste sentido o sucesso da terapêutica dietética para perda de peso depende, em certa medida de tais fatores.

\section{PALAVRAS-CHAVE}

Abandono de terapêutica, Obesidade, Perda de peso, Satisfação com a terapêutica dietética, Suporte social e excesso de peso

ABSTRACT

INTRODUCTION: Overweight has reached epidemic proportions worldwide, and other chronic non-communicable diseases emerge. In this context, there is an increase in the demand for dietary therapy for weight loss, which is not always successful due to low adherence and consequent abandonment of these therapies.

OBJECTIVES: To investigate the probability of abandoning dietary therapy for weight loss according to psychosocial, socioeconomic, demographic, anthropometric and clinical aspects.

METHODOLOGY: Retrospective quantitative cross-sectional study. Developed in two health institutions and one teaching institution. Including adults from 20 to 60 years who had already undergone dietary therapy for weight loss. Questionnaires were applied to collect psychosocial, socioeconomic, demographic, anthropometric and clinical data. Based on the collected data, a model was estimated using the Probit method of multiple regression to investigate which variables influence the probability of abandoning dietary therapy for weight loss.

RESULTS: The sample consisted of 86 individuals, $57 \%$ of whom had abandoned dietary treatment for weight loss. The estimation of the model showed that the educational variable increases the abandonment of dietary therapy for weight loss by 18.9 percentage points. In relation to psychosocial factors, the increase of a degree in satisfaction with therapy and in social support reduces the probability of dropping out by 7.8 percentage points and 5.7 percentage points, respectively. And the variables of a clinical, economic, demographic and anthropometric character were not statistically significant.

CONCLUSIONS: It was found that a high level of education favors the abandonment of dietary therapy for weight loss and satisfaction and social support work in the opposite way to reduce abandonment, in this sense the success of dietary therapy for weight loss depends to some extent of such factors.

KEYWORDS

Abandonment of therapy, Obesity, Weight loss, Satisfaction with diet therapy, Social support and overweight 


\section{INTRODUÇÃO}

O excesso de peso, que abrange a obesidade e o sobrepeso, é definido como o acúmulo excessivo de gordura corporal associado a riscos para a saúde estando associado ao excesso de consumo de calorias em relação ao gasto de energia. Atualmente emerge como importante problema de saúde pública mundial (1) e, em particular, no Brasil. De acordo com dados do Sistema de Vigilância de Fatores de Risco e Proteção para Doenças Crónicas por Inquérito Telefónico/ Vigitel 2019 (2), o excesso de peso, no Brasil, cresceu, em treze anos, passando de 42,6\% em 2006 para 55,4\% em 2019. Ao mesmo tempo a obesidade aumentou no mesmo período de 11,8\% para 20,3\%. Segundo a World Health Organization (WHO), a obesidade é responsável direta e indiretamente por $68 \%$ das mortes no mundo (1). A etiologia da obesidade é complexa envolvendo fatores genéticos, psicológicos, ambientais sociais e económicos (3), de modo que a sua reversão depende em larga medida de mudança comportamental, a qual pode ser facilitada por terapêuticas dietéticas para a perda de peso (TDPP) (4). Todavia, a mudança efetiva de comportamento não é algo trivial (4) associando-se a uma baixa adesão à TDPP, um dos fatores que explicam os pífios resultados destas (5).

Nesta perspetiva muitos estudos vêm investigando as causas dos problemas de adesão e dos fracassos das TDPP, enfatizando as dificuldades para a concretização das mudanças necessárias de hábitos alimentares e de estilo de vida (6-8).

A literatura, todavia, não tem explorado a força de fatores psicossociais, socioeconómicos, clínicos e demográficos no processo de abandono de TDPP, lacuna que o presente estudo visa preencher investigando a probabilidade de abandono segundo características psicossociais, demográficas, socioeconómicas e clínicas.

\section{METODOLOGIA}

Delineamento do Estudo, Local, Questionário e Coleta de Dados

Foi desenvolvido um estudo transversal quantitativo retrospetivo, com base em dados primários. Essa pesquisa foi aprovada pelo Comité de Ética da Faculdade de Ciências Farmacêuticas da Universidade de São Paulo, sob o número 82589718.7.0000.0067.

A pesquisa foi desenvolvida em duas instituições de saúde (um ambulatório de um hospital e uma clínica de nutrição) e em uma instituição de ensino, localizados na cidade de São Paulo/Brasil.

Foram critérios de inclusão: adultos de 20 a 60 anos, de ambos os sexos que, independente do seu estado nutricional, já tivessem se submetido à terapêutica dietética para a perda de peso.

O tamanho da amostra (de conveniência) foi determinado a partir de parâmetro relativo ao Índice de Massa Corporal (IMC) extraído da amostra do estudo piloto com base na fórmula Snedecor e col. em 1967 (9):

$N=\left[\left(z a / 2{ }^{*} \sigma\right) / E\right] 2$

Onde:

$\mathrm{N}=$ é o tamanho da amostra;

za/2 = valor crítico que corresponde ao grau de significância $a=5 \%$; $\sigma=$ Desvio padrão populacional da variável estudada (IMC);

$\mathrm{E}=$ margem de erro admitida

A amostra planejada foi de 90 indivíduos, todavia participaram do estudo apenas 86 em função de dificuldades no recrutamento.

Para o recrutamento, a pesquisa foi divulgada por meio de cartaz, exposto por 4 meses, convidando interessados, para assistir a uma palestra e participar da pesquisa. À medida que se formavam grupos de 20 pessoas, as palestras eram agendadas e realizadas, seguidas da coleta dos dados, por meio da aplicação de dois questionários às pessoas que assinaram o Termo de Consentimento Livre e Esclarecido. O primeiro mediu o grau de suporte social, de motivação e de satisfação com a terapêutica dietética, em duas versões, uma para cada grupo em que foram distribuídos os participantes; indivíduos que abandonaram suas terapêuticas para perda de peso (grupo ABT- abandono de terapêutica) e indivíduos que não abandonaram (grupo TTA- terapêutica), e foi também usado para registrar a avaliação antropométrica. O segundo, mensurou o nível socioeconómico dos participantes.

O primeiro questionário continha questões extraídas e adaptadas de quatro instrumentos, já existentes na literatura nacional e internacional. Os questionários que investigaram fatores intervenientes em terapêuticas dietéticas e adesão à reeducação alimentar $(5,10)$, inspiraram as perguntas relacionadas à satisfação com a terapêutica, tais como: qual foi o seu grau de satisfação com relação à abordagem e ao plano alimentar prescritos pelo profissional. Os aspectos motivacionais foram incluídos a partir do instrumento Treatment SelfRegulation Questionnaires Tobacco (TSRQ tobacco) (11), que analisou as motivações para abandonar o hábito de fumar. Dois dos seus itens adaptados ficaram: perder peso é a melhor maneira de me ajudar; tenho grande vontade de emagrecer e me sentir saudável. E os aspetos ligados a apoio familiar foram considerados com base no APGAR $^{1}$ (12) utilizado para investigação da funcionalidade familiar. Como exemplo de afirmações utilizadas desse instrumento: tenho o apoio e o estímulo de meus familiares para dar continuidade ao meu tratamento; minha família também aderiu às mudanças na alimentação propostas pelo profissional.

O primeiro questionário foi dividido em três partes; a primeira contemplando questões fechadas sobre os aspetos clínicos ${ }^{2}$, demográficos e antropométricos, a segunda abrangendo indagações relativas à satisfação com a terapêutica e a terceira referente ao suporte social e às motivações para a permanência na mesma. As respostas foram avaliadas com base em uma escala Likert. No que tange à satisfação utilizou-se 0 (extremamente insatisfeito) a 4 (extremamente satisfeito), já em relação ao suporte social e motivação, 0 para totalmente falso e 4 totalmente verdadeiro. A avaliação antropométrica foi feita por meio da mensuração de peso e estatura auto referida.

Para a avaliação dos aspectos socioeconómicos (o segundo questionário) foi utilizado o instrumento brasileiro consagrado da Associação Brasileira de Empresas de Pesquisas (13). Este instrumento estima o poder de compra das pessoas, classificando-as em sete classes económicas: A (a mais alta), B1, B2, C1, C2, D e E (a mais baixa), com base no grau de instrução do chefe da família e na quantidade de bens possuídos.

\section{Análise dos Dados}

Para caracterização da amostra e avaliação da homogeneidade dos dois grupos foram utilizadas estatísticas descritivas e testes de igualdade de média/mediana e proporções ${ }^{3}$. Adotou-se como referência o valor de $p<0,050$.

A modelagem da regressão multivariada foi feita pelo método stepwise forward, que consiste em uma análise univariada com cada variável independente para a qual se adota $p<0,200$, com o objetivo de uma pré-seleção. Com base nas variáveis pré-selecionadas foi composto o modelo final com 11 variáveis para estimar a probabilidade de

Adaptation, Partnership, Growth, Affection, Resolve.

${ }^{2}$ Como dados clínicos consideraram-se as seguintes variáveis: utilização de medicamentos, duração da terapêutica, tempo com excesso de peso e tempo tentando perder peso.

${ }^{3} \mathrm{~A}$ distribuição das variáveis foi avaliada por meio do teste de Jarque-Bera, aplicando-se o teste $t$ de student para a diferença de médias no caso de variáveis com distribuição normal e o de Mann Whitney Wilcoxon (Wilcoxon, 1945; Mann e Whitney, 1947) para medianas no caso das variáveis com distribuição não normal. 
abandono de terapêutica dietética para perda de peso por meio do método Probit de regressão múltipla (14), conforme função a seguir. $A B T=f(E s t S a u, I M C, I M C 2$, SupC, Medic, GSAT, GSSOC, GMOTIE, Dtmeses, Epmeses, QTPPmeses)

Onde: $\mathrm{ABT}=$ Abandono (variável binária: $0=$ não abandonou a terapêutica e 1= abandonou a terapêutica); EstSau = variável binária que indica pacientes recrutados em estabelecimentos de saúde $=$ 1, ou em estabelecimento educacional = $0 ; I M C$ = Índice de Massa Corporal; IMC2 = Índice de Massa Corporal ao quadrado; SupC = Nível de Escolaridade (variável binária, menos que superior completo=0, superior completo ou mais $=1$ ); Medic = utilização de medicamento (variável binária, não utiliza $=0$, utiliza=1); GSAT = Grau de Satisfação com a Terapêutica (pontuação de 0 a 32, normalizada na base 10); GSSOC = Grau de Suporte Social (pontuação de 0 a 12, normalizada na base 10);GMOTIE = Grau de Motivação (pontuação de 0 a 80, normalizada na base 10); Dtmeses = Duração da terapêutica (em meses); Epmeses = Tempo com excesso de peso ${ }^{4}$ (em meses); QTPPmeses = Tempo tentando perder peso ${ }^{5}$ (em meses).

A estimação foi realizada por meio do Software StataCorp 2015, adotando-se valor de $p<0,050$.

${ }^{4}$ Entende-se "tempo com excesso de peso", o tempo em que o indivíduo está ou esteve nesta condição.

${ }^{5}$ Entende-se "tempo tentando perder peso", o tempo em que o indivíduo buscou alguma terapêutica para perder peso, que não necessariamente coincide com o tempo com excesso de peso.

\section{RESULTADOS}

\section{Caracterização da População de Estudo}

$\mathrm{Na}$ amostra final foram observados $57 \%$ de indivíduos compondo o grupo que abandonou a terapêutica (ABT) e o restante $43 \%$, integraram o grupo terapêutica (TTA).

Na Tabela 1, observam-se diferenças estatísticas significativas em relação ao uso de medicamentos $(p=0,010)$, escolaridade $(p=0,020)$ e nível socioeconómico $(p=0,050)$.

\section{A probabilidade de Abandono da Terapêutica}

A Tabela 2 apresenta os coeficientes estimados pelo método Probit, que mostram que quanto maior a escolaridade $(p=0,020)$ maior a probabilidade de abandono da TDPP.

No que diz respeito ao grau de satisfação com a terapêutica, evidenciou-se que quanto maior a satisfação, menor a probabilidade de abandono $(p=0,001)$. O mesmo sentido foi obtido em relação ao suporte social, $(p=0,010)$. Já a influência da motivação não foi estatisticamente significativa $(p=0,140)$, ainda que tenha apresentado o sinal negativo esperado. As demais variáveis não foram estatisticamente significativas.

Os resultados do método Probit não indicam a magnitude dos efeitos de cada variável independente sobre a variável dependente (14). Para obter esse impacto foi necessário calcular os efeitos marginais, apresentados na Tabela 3.

\section{Tabela 1}

Caracterização da amostra segundo os grupos abandono e terapêutica e sexo, idade média, nível de escolaridade, Índice de Massa Corporal médio, estado conjugal, utilização de medicamento e classificação socioeconómica

\begin{tabular}{|c|c|c|c|c|c|c|}
\hline & \multicolumn{2}{|r|}{ ABT } & \multicolumn{3}{|c|}{ TTA } & TOTAL \\
\hline & N & IDADE/(MÉDIA) & $\mathbf{N}$ & IDADE/(MÉDIA) & N & IDADE/(MÉDIA) \\
\hline \multicolumn{7}{|l|}{ Sexo/idade média } \\
\hline Mulheres & 37 & 39,1 & 27 & 37,9 & 64 & 38,6 \\
\hline Homens & 12 & 44,0 & 10 & 40,5 & 22 & 42,4 \\
\hline \multirow[t]{3}{*}{ Total Geral } & 49 & 40,5 & 37 & 38,6 & 86 & 39,6 \\
\hline & & ABT & & TTA & & TOTAL \\
\hline & $\mathrm{N}$ & IMC/(MÉDIO) & $\mathbf{N}$ & IMC/(MÉDIO) & $\mathrm{N}$ & IMC/(MÉDIO) \\
\hline \multicolumn{7}{|l|}{ Nível de escolaridade/IMC médio } \\
\hline Superior incompleto ou menos & 12 & 31,6 & 17 & 32,2 & 29 & 31,7 \\
\hline Superior completo ou mais $\left({ }^{\star \star}\right)$ & 37 & 31,5 & 20 & 32,2 & 57 & 31,5 \\
\hline \multirow[t]{3}{*}{ Total geral } & 49 & 30,8 & 37 & 32,6 & 86 & 31,6 \\
\hline & & ABT & & TTA & & TOTAL \\
\hline & N & $\%$ & $\mathbf{N}$ & $\%$ & $\mathbf{N}$ & $\%$ \\
\hline \multicolumn{7}{|l|}{ Estado Conjugal } \\
\hline União estável & 28 & 57,1 & 22 & 59,5 & 50 & 58,1 \\
\hline Fora da união estável & 21 & 42,9 & 15 & 40,5 & 36 & 41,9 \\
\hline Total Geral & 49 & 100,0 & 37 & 100,0 & 86 & 100,0 \\
\hline \multicolumn{7}{|l|}{ Utilização de medicamento } \\
\hline Não utilizou medicamento & 29 & 59,2 & 31 & 83,8 & 60 & 69,8 \\
\hline Utilizou medicamento $\left({ }^{*}\right)$ & 20 & 40,8 & 6 & 16,2 & 26 & 30,2 \\
\hline Total geral & 49 & 100,0 & 37 & 100,0 & 86 & 100,0 \\
\hline \multicolumn{7}{|l|}{ Classe socioeconómica } \\
\hline A & 8 & 16,3 & 8 & 21,6 & 16 & 18,6 \\
\hline B1 & 21 & 42,9 & 8 & 21,6 & 29 & 33,7 \\
\hline B2 & 17 & 34,7 & 18 & $48,6\left({ }^{(\star}\right)$ & 35 & 40,7 \\
\hline $\mathrm{C} 1$ & 3 & 6,1 & 3 & 8,1 & 6 & 7,0 \\
\hline Total Geral & 49 & 100,0 & 37 & 100,0 & 86 & 100,0 \\
\hline $\begin{array}{l}\left.\text { (*) } p<0,010^{(\star \star}\right) p<0,050 \\
\text { ABT: Abandono de terapêutica }\end{array}$ & & & \multicolumn{4}{|c|}{$\begin{array}{l}\text { IMC: Índice de Massa Corporal } \\
\text { N: Número de observações } \\
\text { TTA: Terapêutica }\end{array}$} \\
\hline
\end{tabular}


Tabela 2

Coeficientes Estimados segundo o Modelo Probit para a Probabilidade de Abandono de terapêutica dietética para perda de peso

\begin{tabular}{|c|c|c|c|c|c|c|}
\hline VARIÁVEIS & COEFICIENTE & ERRO PADRÃO & z & p & \multicolumn{2}{|c|}{ IC (90\%) } \\
\hline Estabelecimento de saúde & $-1,78$ & 0,99 & $-1,79$ & 0,070 & $-3,41$ & $-0,14$ \\
\hline Superior completo & 1,05 & 0,43 & 2,42 & 0,020 & 0,34 & 1,76 \\
\hline Medicamento & 0,78 & 0,46 & 1,71 & 0,090 & 0,03 & 1,54 \\
\hline Grau de satisfação & $-0,26$ & 0,09 & $-3,01$ & $<0,001$ & $-0,41$ & $-0,12$ \\
\hline Grau de suporte social & $-0,19$ & 0,07 & $-2,67$ & 0,010 & $-0,31$ & $-0,07$ \\
\hline Grau de motivação intrínseca e extrínseca & $-0,17$ & 0,12 & $-1,48$ & 0,140 & $-0,36$ & 0,02 \\
\hline Duração da terapêutica (meses) & $-0,01$ & 0,02 & $-0,40$ & 0,690 & $-0,04$ & 0,03 \\
\hline IMC & $-0,62$ & 0,47 & $-1,31$ & 0,190 & $-1,40$ & 0,16 \\
\hline IMC ao quadrado & 0,01 & 0,01 & 1,21 & 0,230 & $-0,00$ & 0,02 \\
\hline Tempo com excesso de peso (meses) & $-0,00$ & 0,00 & $-0,41$ & 0,680 & $-0,00$ & 0,00 \\
\hline Tempo tentando perder peso (meses) & $-0,00$ & 0,00 & $-0,94$ & 0,350 & $-0,01$ & 0,00 \\
\hline Constante & 15,45 & 8,05 & 1,92 & 0,060 & 2,22 & 28,69 \\
\hline Pseudo $\mathrm{R}^{2}$ & $43,06 \%$ & & & & & \\
\hline LR chi $^{2}$ & 50,62 & & & & & \\
\hline Prob $>\mathrm{chi}^{2}$ & 0,00 & & & & & \\
\hline Observações & 86 & & & & & \\
\hline
\end{tabular}

ABT: abandono de terapêutica

IC (90\%): Intervalo de Confiança de 90\%

\section{Tabela 3}

Efeitos Marginais do Modelo Probit para a Probabilidade de Abandono de terapêutica dietética para perda de peso

\begin{tabular}{lc}
\multicolumn{1}{c}{ VARIÁVEIS } & $\begin{array}{c}\text { VARIACCÃO DA PROBABILIDADE } \\
\text { DE ABT (PP) }\end{array}$ \\
\hline Estabelecimento de saúde & $-62,20$ \\
\hline Superior completo & 18,86 \\
\hline Medicamento & 16,28 \\
\hline Grau de satisfação & $-7,88$ \\
\hline Grau de suporte social & $-5,68$ \\
\hline Grau de motivação intrínseca e extrínseca & $-5,12$ \\
\hline Duração da terapêutica (meses) & -0.26 \\
\hline IMC & $-2,05$ \\
\hline Tempo com excesso de peso (meses) & $-0,03$ \\
\hline Tempo tentando perder peso (meses) & $-0,09$ \\
\hline $\mathbf{N}$ & $\mathbf{8 6}$ \\
\hline
\end{tabular}

ABT: abandono de terapêutica

IMC: Índice de Massa Corporal

$\mathrm{N}$ : tamanho da amostra

pp: pontos percentuais

A partir dos coeficientes estimados e dos respetivos efeitos marginais, constatou-se que o indivíduo referência tinha uma probabilidade de $77,6 \%$ de abandonar sua terapêutica dietética para perda de peso. Essa probabilidade se eleva a 93,9\% para indivíduos com maior nível de escolaridade, mas se reduz para $68,7 \%$ caso possuam um ponto a mais de satisfação e de suporte social do que o indivíduo referência.

\section{DISCUSSÃO DOS RESULTADOS}

Em um cenário de aumento da incidência da obesidade, de estilos de vida conflitantes com as regras básicas do peso adequado; e de oferta abundante de alimentos processados e de equipamentos que poupam as pessoas de gasto energético (15), cabe discutir a força de fatores que possam incentivar ou inibir os comportamentos saudáveis e a persistência em TDPP.

As pessoas com excesso de peso buscam terapêuticas para perda de peso, visando a saúde e a estética. Todavia, o sucesso de tais programas, não é suficientemente frequente $(5,16-18)$.

O sinal positivo encontrado para a escolaridade foi inesperado,
IMC: Índice de Massa Corporal

pois considera-se que o nível de instrução elevado atribui maior capacidade de entendimento sobre a importância das orientações do profissional. Esse resultado, contrário ao esperado, também foi obtido no estudo de caso controle desenhado por Abo e col. em 2016 (19), cujo objetivo foi estudar os fatores que influenciam a não adesão aos regimes alimentares entre adultos obesos em Tanta, Egito. De facto, indivíduos com maior nível intelectual podem sentir que não precisam de acompanhamento periódico de um profissional, por acreditarem estar aptos a seguir por conta própria as mudanças alimentares necessárias.

No que concerne aos aspetos psicossociais, a baixa satisfação aumenta a probabilidade de abandono de terapêutica dietoterápica para perda de peso. Neste contexto destaca-se o estudo de revisão sistemática de Greavesa e col. em 2017 (20), entre outros, sobre manutenção de peso em adultos, com a conclusão de que a perda e o controle de peso bem-sucedidos envolvem, entre outros fatores, a satisfação com a terapêutica e o apoio do profissional que o assistiu. A conduta do nutricionista é um elemento básico para a satisfação do paciente com sua terapêutica. A humanização no serviço de saúde, com a criação de um atendimento baseado no diálogo, no entendimento e no auxílio às dificuldades do indivíduo, como também a empatia e a escuta são fundamentais neste processo. A convicção do paciente em seu tratamento tem como primeira instância a confiança no profissional e na sua abordagem (17)

O desenvolvimento do vínculo entre profissional e paciente tem sido cada vez mais valorizado, e por sua vez, têm conexão positiva com a adesão à terapêutica. Estudo brasileiro desenvolvido com o objetivo de analisar a potencialidade da abordagem grupal, utilizando-se de estratégias de educação alimentar e nutricional, apontou que o resultado do tratamento é consequência da interação entre paciente e nutricionista, e esta decorre da atuação do profissional, entretanto o protagonismo para promover essa interação depende da habilidade do mesmo em conduzir o processo de mudanças comportamentais (21).

A importância da satisfação com a TDPP, evidenciada no presente estudo, vai também ao encontro dos achados da pesquisa de Oliveira e col. em 2014 (22), na qual analisaram a satisfação com o 
atendimento de pacientes em uma Clínica de Nutrição da PUC de Minas Gerais/Br, concluindo que essa variável impacta diretamente a decisão de permanência ou não do paciente na terapêutica.

Em relação ao suporte social, o acolhimento percebido, tanto do grupo familiar ou de amigos, quanto do próprio profissional de saúde, leva o paciente a ter uma maior segurança e força para continuar seguindo seu objetivo $(16,18,8,23)$. A importância do papel do nutricionista e da família no contexto de terapêutica também foi referenciada em estudo de Cangerana Santiago e col. em 2015 (24), cujo objetivo foi entender quais fatores levam ao abandono de tratamento nutricional para perda de peso de crianças com excesso de peso, concluiu-se que a falta de vínculo e de empatia do profissional assim como a falta de apoio do grupo familiar são fatores predisponentes ao abandono. Estudos como os de Varkevisser e col. em 2019 (16) e Taglietti e col. em 2018 (17), os quais tiveram por objetivo, entender os determinantes para a perda de peso e descrever os aspetos subjetivos do tratamento para redução de peso, respetivamente, mostram que as motivações têm importância para a continuidade do tratamento, mas somente esta força de vontade não é suficiente, como demonstrado no presente estudo. Como ponto forte deste trabalho destaca-se o embasamento econométrico do estudo que permitiu estimar a probabilidade de abandono e o papel de fatores psicossociais sobre essa probabilidade, demonstrando a importância desses fatores para a continuidade dos TDPP. As limitações do estudo derivam da forma de recrutamento, da amostragem por conveniência e do tamanho da amostra inferior ao planejado, que podem ter gerado viés de seleção. Nesse sentido, considera-se que novos estudos são necessários para aumentar a compreensão de tema tão complexo.

\section{CONCLUSÕES}

Verificou-se que os fatores psicossociais como satisfação com a terapêutica e suporte social influenciam favoravelmente, reduzindo a probabilidade de abandono. Além desses, o nível elevado de escolaridade, por outro lado, atuou em sentido contrário, favorecendo o abandono de TDPP. Os efeitos das demais variáveis testadas, de caráter clínico, económico, demográfico e antropométrico não foram estatisticamente significativas.

Diante destas constatações, evidencia-se a importância do papel do nutricionista responsável pelo atendimento do indivíduo com excesso de peso, e do tipo de abordagem, onde a humanização e a empatia se destaquem. O apoio social e da família também tem um papel de grande importância no processo para perda de peso, cabendo ao nutricionista identificar a qualidade de tais apoios e adotar uma terapia holística envolvendo outros tipos de profissionais como psicólogos e assistentes sociais.

Neste contexto, estratégias de atendimento que priorizem tais evidências podem aumentar as chances de sucesso das terapêuticas para perda de peso.

\section{REFERÊNCIAS BIBLIOGRÁFICAS}

1. World Health Organization. Global status report on noncommunicable diseases 2014. Geneva: World Health Organization; 2015.

2. Brasil. Ministério da Saúde. Vigitel Brasil 2019: vigilância de fatores de risco e proteção para doenças crônicas por inquérito telefônico: estimativas sobre frequência e distribuição sociodemográfica de fatores de risco e proteção para doenças crônicas nas capitais dos 26 estados brasileiros e no Distrito Federal em 2019 [recurso eletrônico] / Ministério da Saúde, Secretaria de Vigilância em Saúde, Departamento de Análise em Saúde e Vigilância de Doenças não Transmissíveis. - Brasília: Ministério da Saúde, 2020. 137.: il. (Acessado em 05 de Julho de 2020). Disponível em: http:// bvsms.saude.gov.br/bvs/publicacoes/vigitel_brasil_2019_vigilancia_fatores_risco.pdf.
3. Agência Nacional de Saúde Suplementar (Brasil). Diretoria de Normas e Habilitação dos Produtos. Gerência-Geral de Regulação Assistencial. Gerência de Monitoramento Assistencial. Coordenadoria de Informações Assistenciais. Manual de diretrizes para o enfretamento da obesidade na saúde suplementar brasileira [recurso eletrônico]. Rio de Janeiro: ANS, 2017.

4. Botchlett R, Woo SL, Liu M, Pei Y, Guo X, Li H, Wu C. Nutritional Approaches for Managing Obesity-Associated Metabolic Diseases. J Endocrinol. 2017; 233(3): R145-R171.

5. Gonçalves ISA, Ferreira NTMY, Reis PVS, Pena GG. Fatores intervenientes no seguimento do tratamento nutricional para redução de peso em mulheres atendidas em uma unidade básica de saúde do Brasil. Revista Cuidarte. 2015; 6(1): 914-922. 6. Lindemann IL, Oliveira RR, Mendoza-Sassi RA. Dificuldades para alimentação saudável entre usuários da atenção básica em saúde e fatores associados. Ciência e Saúde coletiva. 2016; 2(2): 599-610.

7. Halali F, Lapveteläinen A, Karhunen L, Saarela AM, Lappalainen R, Kantanen T. Motivators, barriers and strategies of weight management: A cross-sectional study among Finnish adults. Eating Behaviors. 2018; 31: 80-87.

8. Shields AT. Examination of the obesity epidemic from a behavioral perspective. International Journal of Behavioral and Consultation Therapy. 2009; 5(1): 142-158.

9. Snedecor GW, Cochran WG. Statistical methods. $6^{a}$ edição. Ames, Lowa, the Lowa state University. 1967.

10. Koehlein EA, Salado GA, Yamada AN. Adesão à reeducação alimentar para perda de peso: determinantes, resultados e percepção do paciente. Revista Brasileira de Nutrição Clínica. 2008; 23(1): 58-65.

11. Levesque CS, Williams GC, Elliot D, Pickering MA, Bodenhamer B, Finley PJ. Validating the theoretical structure of the treatment self-regulation questionnaire (TSRQ) across three different health behaviors. Health Education Research. 2007; 22(5): 691-702. 12. Duarte YAO. Família: rede de suporte ou fator estressor: a ótica de idosos e cuidadores familiares São Paulo. Escola de Enfermagem. Universidade de São Paulo. 2001. Tese. 13. Associação Brasileira de Empresas de Pesquisa - ABEP. 2015.

14. Wooldridge, JM. Introdução a Econometria. $4^{a}$ edição. São Paulo: Cengage Learning, 2010.

15. Alvarenga M. Fundamentos teóricos sobre análise e mudança de comportamento. In: Alvarenga M, Figueiredo M, Timermam F, Antonaccio C. Nutrição Comportamental. $1^{a}$ edição. São Paulo: Editora Manole. 2015.

16. Varkevisser RDM, Van Stralen MM, Kroeze W, Ket JCF, Steenhuis IHM. Determinants of weight loss maintenance: a systematic review Obesity reviews. 2019; 20:171-211. 17. Taglietti RL, Riepe SB, Maronezi TB, Teo CRPA. Tratamento nutricional para redução de peso: aspectos subjetivos do processo. Revista Brasileira de Obesidade, Nutrição e Emagrecimento. 2018; 12(69): 101-109.

18. Oliveira APSV, Silva MM. Fatores que dificultam a perda de peso em mulheres obesas de graus I e II. Revista Psicologia e Saúde. 2014; 6(1): 74-82.

19. Abo ALI, Ehab A, Atlam Salwa A, Ghareeb Wessam A. Findings from Tanta University Update Understanding of Obesity (Factors behind nonadherence to diet regimens among obese adults in Tanta, Egypt: a case-control study). The Journal of the Egyptian Public Health Association. 2016; 91(1): 8-14.

20. Greavesa C, Poltawskia L, Garsideb R, Briscoec S. Understanding the challenge of weight loss maintenance: a systematic review and synthesis of qualitative research on weight loss maintenance. Health Psychology Review, 2017; 11(2): 145-163.

21. Vincha KRR, Bógus CM, Cervato-Mancuso AM. Possibilidades de atuação profissional em grupos educativos de alimentação e nutrição. Interface (Botucatu). 2020; 24: e190028.

22. Oliveira TRPR, Pereira CG. Perfil de pacientes que procuram a clínica de nutrição da PUC- Minas e satisfação quanto ao atendimento. Percurso Acadêmico. 2014; 4(8): 268-282. 23. Simpson SA, Matthews L, Pugmire J, McConnachie A, Mclntosh E, Coulman E, Hughes K, Kelson M, Morgan-Trimmer S, Murphy S, Utkina-Macaskill O, Moore LAR. An app-, weband social support-based weight loss intervention for adults with obesity: the 'HelpMeDolt!' feasibility randomised controlled trial. Pilot and Feasibility Studies. 2020; 6(133): 1-14. 24. Cangerana Santiago LA, De Nóbrega FJ. Elaboração de protocolo para atendimento nutricional de crianças obesas ou com sobrepeso. Journal of Health \& Biological Sciences. 2015; 3(3): 151-158. 